\title{
可见光促进的苄位 $\mathrm{C}_{\mathrm{sp} 3}$ - $\mathrm{H}$ 键活化官能团化反应
}

\author{
裴朋昆张凡易红雷爱文* \\ (武汉大学化学与分子科学学院 武汉 430072)
}

\begin{abstract}
摘要 近年来, 可见光促进的光致氧化还原催化活化有机小分子在合成化学中蓬勃发展. 该策略一般以过渡金属有机 络合物或有机染料作为光敏剂, 在可见光照射下, 其通过与底物发生单电子转移(SET)发生相互作用, 利用反应过程中 形成的自由基或自由基离子中间体，实现 $\mathrm{C}-\mathrm{X}(\mathrm{X}=\mathrm{C}, \mathrm{N}, \mathrm{O} \ldots \ldots)$ 键节能高效、高选择性、绿色性构建. 本文根据不同 的关键反应中间体, 就可见光促进的苄位 $\mathrm{C}_{\mathrm{sp} 3}-\mathrm{H}$ 键活化官能团化近年来取得进展进行简要归总.
\end{abstract}

关键词 可见光催化; $\mathrm{C}_{\mathrm{sp} 3}-\mathrm{H}$ 键活化; 甲苯类衍生物; 绿色化学

\section{Visible Light Promoted Benzylic $\mathrm{C}_{\mathrm{sp} 3}-\mathrm{H}$ Bond Activation and Functionalization}

\author{
Pei, Pengkun Z Zhang, Fan Yi, Hong Lei, Aiwen* \\ (College of Chemistry and Molecular Sciences, Wuhan University, Wuhan 430072)
}

\begin{abstract}
In recent years, visible-light-promoted photoredox catalytic activation of organic molecules has been flourishing vigorously. This kind of methodology usually takes advantage of transition-metal complexes and organic dyes as photosensitizers, which can directly react with organic substrates through a single-electron-transfer (SET) progress under visible light irradiation. It's operable to construct $\mathrm{C}-\mathrm{X}(\mathrm{X}=\mathrm{C}, \mathrm{N}, \mathrm{O} \ldots)$ bond via the radical or radical ion generated during the SET process. On the basis of different key intermediates, this highlight gives a brief summary on the recent development of visible light promoted benzylic $\mathrm{C}_{\mathrm{sp} 3}-\mathrm{H}$ activation and functionalization.
\end{abstract}

Keywords visible light photoredox; $\mathrm{C}_{\mathrm{sp} 3}-\mathrm{H}$ bond activation; toluene derivatives; green chemistry

\section{1 引言}

探寻实现高效性、高选择性、高经济性、绿色、安 全的合成策略是化学工作者们孜孜不倦的追求. 新型成 键模式的不断涌现使方法学在药物、天然产品、超分子、 商业化试剂等领域的开发应用中存在巨大的潜力和价 值. 而直接使用广泛存在的碳氢化合物作为合成子, 实 现 $\mathrm{C}-\mathrm{H}$ 键到 $\mathrm{C}-\mathrm{X}$ 键 $(\mathrm{X}=\mathrm{C}, \mathrm{N}, \mathrm{O} \ldots \ldots)$ 的转化无疑更 具诱惑力和挑战性. 甲苯类衍生物, 作为一类廉价易得 的工业原料, 其苄位 $\mathrm{C}_{\mathrm{sp} 3}-\mathrm{H}$ 键选择性活化官能团化也 一直是科学研究的热门领域 ${ }^{[1]}$.

催化领域一个重要的目标就是寻找新方法来活化 小分子, 其中光催化作为一种新手段近来受到很多关 注 $^{[2]}$. 光催化反应的实验过程中可以使用常规的玻璃仪 器 ${ }^{[3]}$, 无需加热和腐蚀性化学品 ${ }^{[4]}$, 底物大都也不需要 预先官能团化, 且多数反应可以在温和的反应条件下高
效进行，无疑在本质上更具优势 ${ }^{[5]}$. 然而大多数有机物 本身并不感光，因此多种光敏剂被用来吸收光子以活化 体系中的有机基质. 在可见光的照射下, 受激发的电子 从 HOMO 跃迁到 LUMO, 再经过系统间交换形成相对 寿命较长的三重激发态, 其相较于基态物质既是更强的 氧化剂也是更强的还原剂, 继而可发生还原淬灭或氧化 淬灭 ${ }^{[6]}$. 其单电子氧化还原历程也被化学家用来设计新 型有机化学反应 ${ }^{[7]}$. 可见光催化由于其低成本、储量丰 富、绿色无毒 ${ }^{[8]}$ 、可再生 ${ }^{[9]}$ 等特点, 近年来在有机合成领 域得到了迅猛发展，成为一种重要的有机合成工具 ${ }^{[10]}$. 不同甲苯类底物通过单电子转移过程可得到不同的苄 位自由基或苄位自由基离子中间体，进而发生多种多样 的反应. 本文依据不同反应活性中间体，对近年来可见 光促进的苄位 $\mathrm{C}_{\mathrm{sp} 3}-\mathrm{H}$ 键活化官能团化反应进行简要归 总(图 1).

\footnotetext{
*E-mail: aiwenlei@whu.edu.cn

Received August 16, 2016; published September 20, 2016.

Project supported by the 973 Program (2012CB725302), the National Natural Science Foundation of China (21390400, 21520102003, 21272180 and 21302148), and the Research Fund for the Doctoral Program of Higher Education of China (20120141130002) and the Program for Changjiang Scholars and Innovative Research Team in University (IRT1030) and the Ministry of Science and Technology of China (2012YQ120060), the Program of Introducing Talents of Discipline to Universities of China (111 Program) is also appreciated.

项目受科技部 973 计划(No. 2012CB725302)、国家自然科学基金(Nos. 21390400, 21520102003, 21272180, 21302148)、高等学校博士学科点专项科研 基金(No. 20120141130002)、教育部长江学者和创新团队发展计划(No. IRT1030)、国家科学技术部基金(No. 2012YQ120060)和高等学校学科创新引智 计划(111 项目)资助.
} 
Benzylic $\mathrm{C}_{\mathrm{sp} 3}-\mathrm{H}$ Activation and Functionalization:

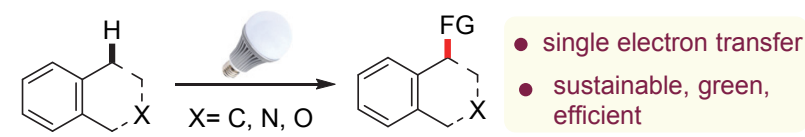

Reaction Intermediate:
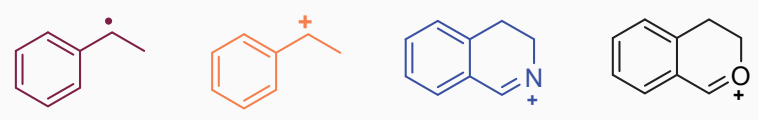

图 1 可见光促进的 $\mathrm{C}_{\mathrm{sp} 3}-\mathrm{H}$ 键活化

Figure 1 Visible light promoted $\mathrm{C}-\mathrm{H}$ bond activation

\section{2 碳自由基介导的苄位 $\mathrm{C}_{\mathrm{sp} 3}-\mathrm{H}$ 键活化官能团 化}

2013 年, 陈教授课题组 ${ }^{[11]}$ 成功地以二芳基酮类化 合物作为光敏剂, 实现了芐位 $\mathrm{C}_{\mathrm{sp} 3}-\mathrm{H}$ 键的活化单氟化 反应, 并通过进一步调节光敏剂活性和篮选氟源首次实 现了苄位偕二甲基的双氟化反应(图 2). 该方法学可以 很好的兼容芳环和支链含有不同富电子和贫电子官能 团 $(-\mathrm{CN},-\mathrm{F},-\mathrm{Cl} \ldots \ldots)$ 的底物. 文章中对取代基的空 间位阻效应给出了详尽阐释. 此反应的动力学同位素效 应(KIE)表明该反应的决速步为非金属自由基的 $\mathrm{C}-\mathrm{H}$ 㩲取过程. 为展现此反应在有机合成中的应用前景, $1 \mathrm{~g}$ 规模的反应也被成功实行. 随后, 该课题组又进一步以 苯乙酮为光促进剂, 实现了㤢性 $\mathrm{C}_{\mathrm{sp} 3}-\mathrm{H}$ 键的活化氟化 反应, 并认为其仅参与㩖氢过程 ${ }^{[12]}$.

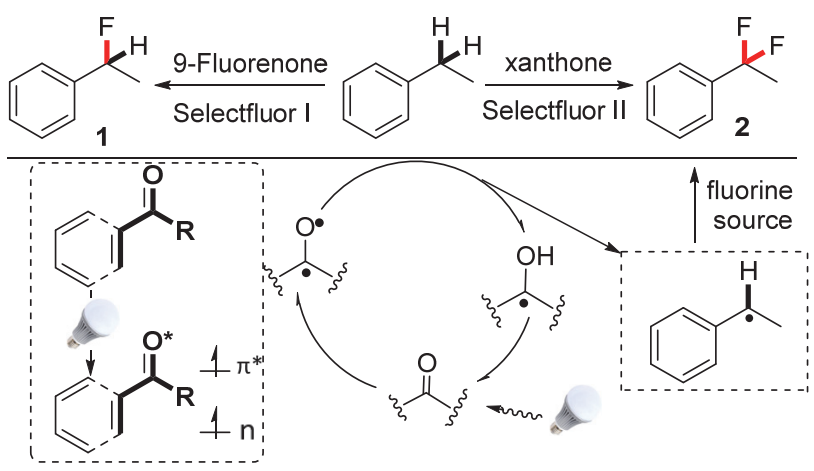

图 2 芳基酮催化的 $\mathrm{C}_{\mathrm{sp} 3}-\mathrm{H}$ 键氟化反应

Figure $2 \mathrm{C}_{\mathrm{sp} 3}-\mathrm{H}$ fluorination catalyzed by arylketones

2013 年, MacMillan 教授课题组 ${ }^{[13]}$ 通过可见光诱导 的有机小分子催化氧化还原策略, 利用碳自由基/碳自 由基偶联, 实现了芐醚 $\mathrm{C}_{\mathrm{sp} 3}-\mathrm{H}$ 键的直接活化芳基化(图 3). 该方法学具有良好的官能团耐受性和底物兼容性, 甚至适用于高度拥挤原子处的 $\mathrm{C}-\mathrm{C}$ 成键. 文章中突出 强调键离解能(BDEs)、氢原子转移(HAT) 常数、氧化还 原电位三个参数的重要性. 需要特别指出的是, 二苄基 醚类反应物能专一地转化为相应的单芳基化产物, 并且 二氢呋喃在该反应条件下也可以高效高立体选择性地 得到目标产物.<smiles>CO[CH+]Cc1ccccc1</smiles>

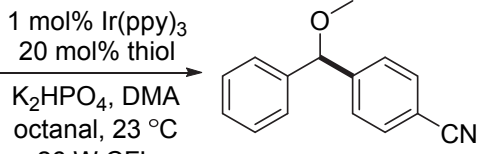
26 W CFL<smiles>COCc1ccccc1</smiles><smiles>COCc1ccccc1CCc1ccc(C#N)cc1</smiles>

Radical-Radical Coupling photoredox $\uparrow$ catalyst<smiles>N#Cc1ccc(C#N)cc1</smiles>

图 3 芐醚的直接芳基化反应

Figure 3 Direct arylation of benzylic ethers

2014 年, MacMillan 课题组 ${ }^{[14]}$ 利用相似的策略, 以 铱/硫醇协同催化苄醚和 Schiff 碱为反应底物的自由基自由基偶联反应构建 $\beta$-氨基醚类化合物(图 4). 比较之 下，富电子的苄基醚以及带电中性基团的亚胺能以更高 的效率完成反应. 他们推测该反应机理可能为: 可见光

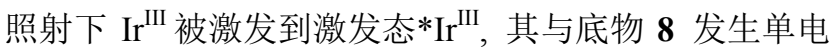
子转移而被还原淬灭并产生自由基阴离子 $\mathbf{9}$; 中间体 $\mathbf{5}$ 进一步与颈基乙酸甲酯发生质子偶联电子转移(PCET) 作用, 生成的硫自由基 7 与茮醚类底物 10 发生氢原子 转移, 实现有机催化小分子再生并产生苄基自由基 11, 由稳态自由基效应可知，自由基 9 与 11 可直接偶联生 成目标产物.
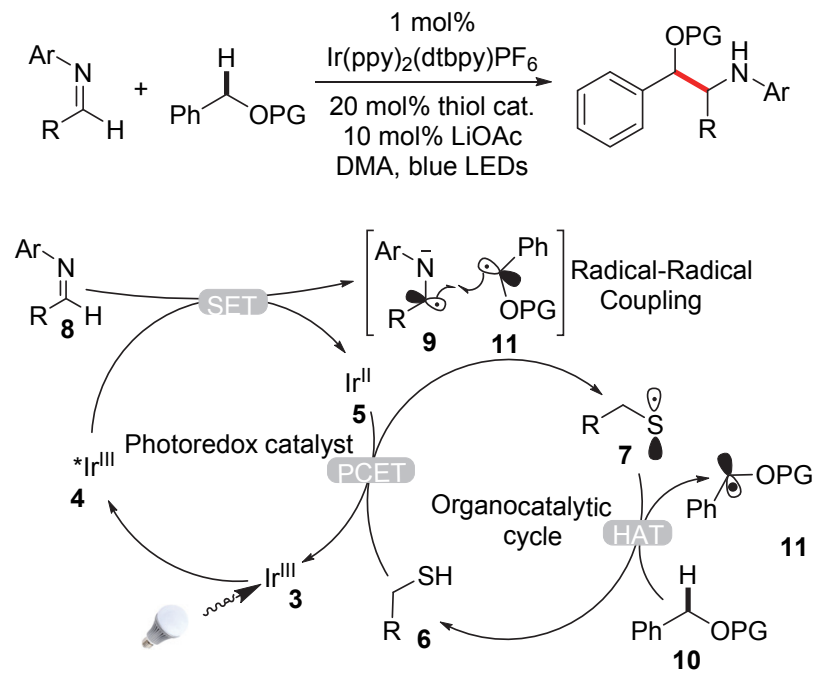

图 4 交叉偶联反应机理

Figure 4 Mechanism of the heterocoupling reaction

2016 年, Greaney 课题组 ${ }^{[15]}$ 以 $\mathrm{Cu}(\mathrm{dap})_{2} \mathrm{Cl}$ 为光促进 剂, 实现了多种甲苯类衍生物苄位 $\mathrm{C}_{\mathrm{sp} 3}-\mathrm{H}$ 键的直接叠 
氮化(图 5), 该反应具有广泛的官能团耐受性, 并可兼容 伯碳、叔碳、季碳类甲苯底物. 他们推测该反应可能是 碳自由基介导的自由基链式反应.
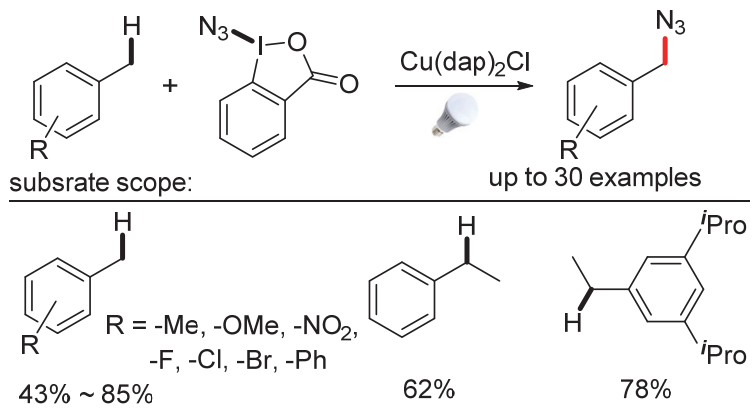

$78 \%$

图 5 芐位叠氮化反应链增殖过程

Figure 5 Radical chain propagation of $\mathrm{C}_{\mathrm{sp} 3}-\mathrm{H}$ azidation

2016 年, 李课题组 ${ }^{[16]}$ 通过 “一锅两步法” 实现了可 见光促进的乙苯类衍生物茮位惰性 $\mathrm{C}_{\mathrm{sp} 3}-\mathrm{H}$ 键活化胺化 反应构建苄胺类化合物(图 6). 其他溴阴离子化合物的 加入未能影响反应率, 并且 $\mathrm{AgF}$ 的加入也未能产生相应 的苄基氟化物. 因此他们认为该反应可能经历了一个碳 自由基介导的链增殖过程. 同样地, 该课题组利用相似 的策略实现了烷基苯基醚到烷酯或烷醇的转化 ${ }^{[17]}$. 通 过机理验证, 表明反应可能经历氧 $\alpha$ 位 $\mathrm{C}_{\mathrm{sp} 3}-\mathrm{H}$ 键均裂 的链增殖过程, 并认为 $\alpha$-溴代醚为反应活性中间体.

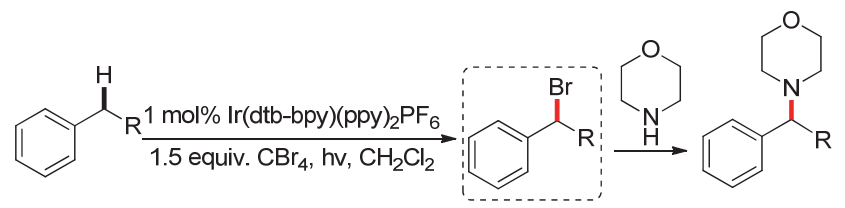

图 6 “一锅法” 构建苄位 C-N 键

Figure 6 One-pot benzylic $\mathrm{C}-\mathrm{N}$ bond formation

\section{3 碳正离子介导的苄位 $\mathrm{C}_{\mathrm{sp} 3}$ - $\mathrm{H}$ 键活化官能团 化}

以碳正离子为反应活性中间体活化芐位 $\mathrm{C}_{\mathrm{sp} 3}-\mathrm{H}$ 键 的报道相对比较少. 2016 年, Pandey 课题组 ${ }^{[18]}$ 以可见光 促进的方式形成碳正离子中间体, 分别以水和二级胺类 底物作为亲核试剂, 高效、高选择性地实现了分子间交 叉脱氢偶联 $(\mathrm{CDC})$ 反应构建 $\mathrm{C}-\mathrm{O} / \mathrm{C}-\mathrm{N}$ 键(图 7),

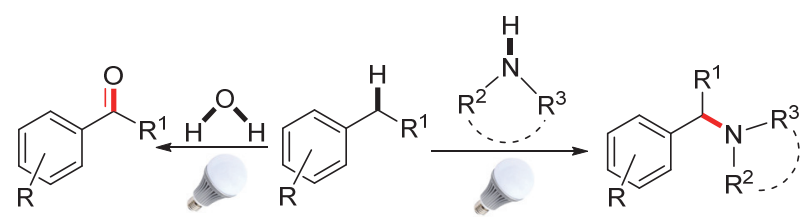

$\mathrm{R}=-\mathrm{OMe},-\mathrm{H},-\mathrm{t}-\mathrm{Bu}$

$\mathrm{R}^{1}=$-alkyl, -aryl, -ester

15 examples

$\mathrm{R}=-\mathrm{OMe},-\mathrm{H}$

$\mathrm{R}^{1}=$-alkyl, -aryl, -ester

28 examples

图 7 苄位 $\mathrm{C}_{\mathrm{sp} 3}-\mathrm{H}$ 键到 $\mathrm{C}-\mathrm{N} / \mathrm{O}$ 键转化

Figure 7 Benzylic $\mathrm{C}_{\mathrm{sp} 3}-\mathrm{H}$ functionalization for $\mathrm{C}-\mathrm{N} / \mathrm{O}$ bond formation
尤其为唑类化合物的修饰提供了一条简单易行的途 径. 通过控制实验，他们验证了相应的醇为反应中间体.

\section{4 亚胺离子介导的芳位 $\mathrm{C}_{\mathrm{sp} 3}$ - $\mathrm{H}$ 键活化官能团 化}

2011 年, 肖文精教授课题组 ${ }^{[19]}$ 利用光敏剂/氧气体 系, 在可见光催化下, 通过串联氧化/[3+2]环加成/氧化 芳构化反应实现了含氮杂环天然产物片螺素的合成(图 8). 该串联反应具有极好的立体选择性，当用非对称性 双偶极物质做反应物时，只有一种位置异构体生成. 氞 代实验表明茮位篗氢过程可能是反应的决速步.
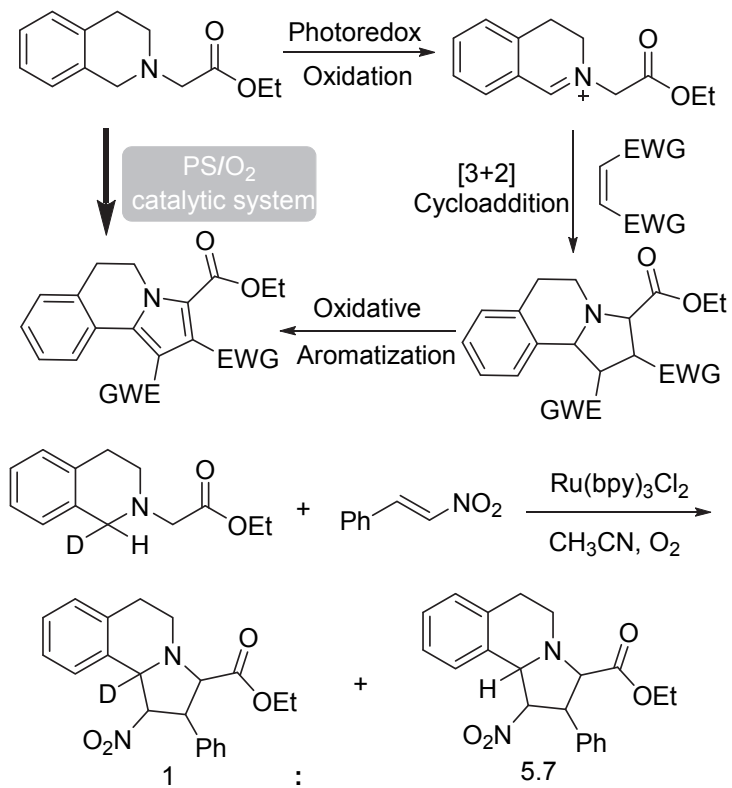

图 8 光催化氧化/环加成/芳构化历程

Figure 8 Photocatalytic oxidation/cycloaddition/aromatization sequence

2012 年, 吴骊珠教授课题组 ${ }^{[20]}$ 在可见光诱导下成 功实现 $N$-芳基取代四氢异喹啉和硝基烷烃类衍生物、丙 二酸二乙酯、丙酮分子间的需氧交叉脱氢偶联(CDC)反 应构建 $\mathrm{C}_{\mathrm{sp} 3}-\mathrm{C}_{\mathrm{sp} 3}$ 键(图 9). 结合瞬态吸收光谱, 电子自 旋共振吸收光谱, UV-Vis 吸收光谱和苂光发射光谱等机 理验证实验，她们首次发现了 $N$-芳基取代四氢异喹啉 到有机光促进剂 TBA-eosin Y 的单电子转移过程, 并认 为反应过程中产生的超氧自由基阴离子而非单线态氧 气对于加速该转化的速率具有重要的作用.

2013 年, 该课题组采用具有更高氧化还原能力的 二价铂络合物代替二联吡啶钌, 在硫酸亚铁的协同作用 下, 首次实现了 $\mathrm{Pt}^{\mathrm{II}}$ 催化的氧气到超氧自由基负离子转 化，完成 $N$-芳基取代四氢异喹啉和吲哚类衍生物的需 氧交叉脱氢偶联反应，同样借助谱学的手段提出了和上 面相似的机理 ${ }^{[21]}$.

较之之前的需氧交叉偶联脱氢反应，虽然交叉脱氢 偶联反应可以很好地避免底物的预官能团化步骤，以氧 


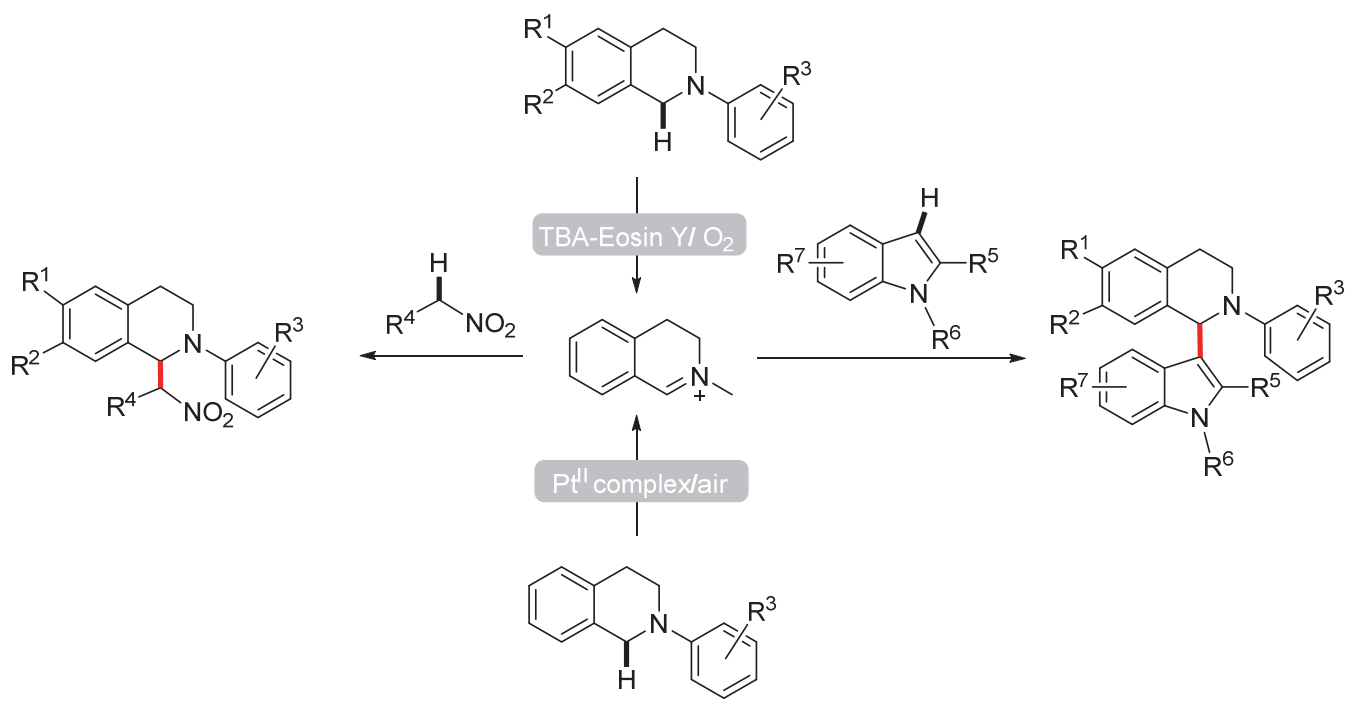

图 9 可见光促进的需氧交叉脱氢偶联可能机理

Figure 9 Aerobic photocatalytic $\mathrm{CDC}$ reactions

气代替当量氧化剂的使用, 并避免当量化学废料的产 生, 而水作为唯一的副产物. 但直接使用不同的 $\mathrm{C}-\mathrm{H}$ 化合物在无额外氧化剂存在的条件下实现其氧化交叉 偶联放氢反应无疑更具原子经济性和绿色性. 吴骊珠教 授课题组 ${ }^{[22 ~ 25]}$ 率先报道了系列质子还原催化剂(Proton Reductive Catalyst, PRC)存在下的光催化产氢体系, 实 现了多重键型的高效、高选择性、清洁、经济构建(图 10).

$$
\mathrm{Nu}-\mathrm{H}+\mathrm{Nu}-\mathrm{H} \underset{\text { visible light }}{\stackrel{\text { oxidant free }}{\longrightarrow}} \mathrm{Nu}-\mathrm{Nu}+\mathrm{H}_{2} \mathrm{O}
$$

图 10 无额外氧化剂存在下交叉偶联放氢反应

Figure 10 Oxidant-free photocatalytic CCHE reaction
2013 年, 吴课题组 ${ }^{[26]}$ 率先报道了无额外氧化剂存 在条件下的交叉偶联放氢反应, 以四氢异喹啉和㗱类 衍生物作为底物, 在曙红和 $\mathrm{G}-\mathrm{RuO}_{2}$ 作为光敏剂的条件 下，实现两种不同 $\mathrm{C}-\mathrm{H}$ 化合物的直接偶联. 反应基质 的强电子效应会对此反应的产率有较大的影响, 并且 $N$-苯基四氢异喹啉 4 号位的强吸电子基完全抑制了反应 的进行. 2016 年, 该课题组 ${ }^{[27]}$ 在之前研究的基础上, 同 样利用 CCHE 策略, 以二价铂络合物同时作为光敏剂和 质子转移催化剂, 实现了三芳基取代吡唑啉类化合物到 吡坐的脱氢转化(图 11). 通过紫外-可见光谱, 苂光光 谱, ${ }^{1} \mathrm{H}$ 核磁谱, 发射光谱以及瞬态吸收光谱等手段, 她 们认为该反应最初是由光引发的单电子转移过程, 随后 质子偶联释放出氢气.

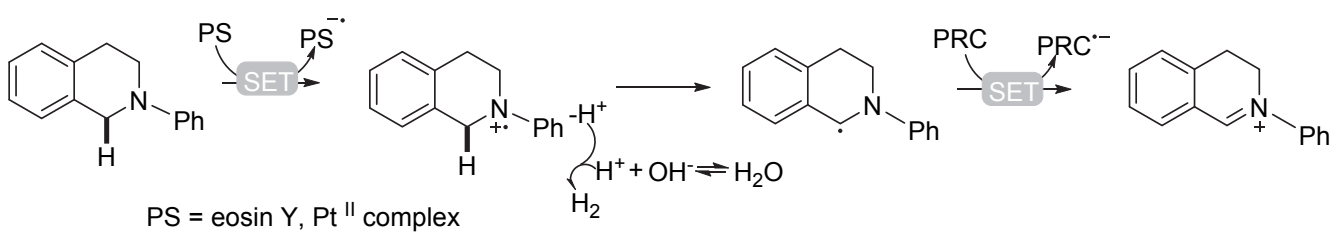
$\mathrm{PRC}=\mathrm{Co}[\mathrm{L}], \mathrm{Pt}{ }^{\prime \prime}$ complex<smiles></smiles>

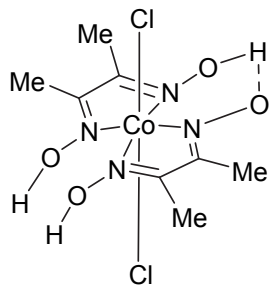

$\mathrm{Co}(\mathrm{dmgH})_{2} \mathrm{Cl}_{2}$

图 11 以亚胺正离子为介导的 $\mathrm{CCHE}$ 反应

Figure 11 Imine cation mediated CCHE reaction 


\section{5 氧鎓离子介导的苄位 $\mathrm{C}_{\mathrm{sp} 3}-\mathrm{H}$ 键活化官能团 化}

相比于氮而言, 氧上的孤对电子更难于被氧化, 因 此以氧鎓离子为介导的光催化 $\mathrm{C}_{\mathrm{sp} 3}-\mathrm{H}$ 键活化屈指可数. 2015 年, 吴骊珠教授课题组在无额外氧化剂存在的条 件下, 通过光敏剂 $\mathrm{Acr}-\mathrm{Mes}^{+} \mathrm{ClO}_{4}{ }^{-}$和质子转移催化剂 $\mathrm{Co}\left(\mathrm{dmgBF}_{2}\right)_{2} \cdot 2 \mathrm{MeCN}$ 的协同作用, 以 $\mathrm{Cu}(\mathrm{oTf})_{2}$ 为添加 剂, 实现了异色满和 $\beta$-酮酸酯分子间的交叉偶联放氢反 应(图 12) ${ }^{[28]}$. 脂肪族和不同电性的芳基 $\beta$-酮酯都能很好 地发生反应, 位阻影响在文中也有详细描述. 辅以瞬态 吸收光谱, 她们推测该反应可能经历了一个光促进剂还 原淬灭的过程, 并形成氧鎓离子中间体, 同时 KIE 实验 表明苄位 $\mathrm{C}-\mathrm{H}$ 键的断裂可能是反应的决速步.

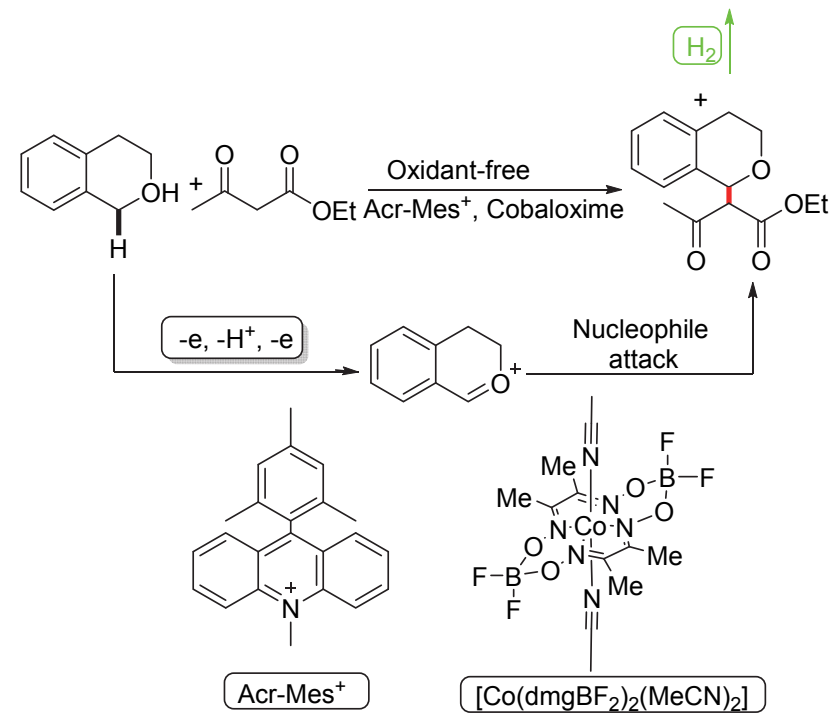

图 12 无额外氧化剂存在下光催化析氢交叉偶联

Figure 12 Oxidant-free photocatalytic cross-coupling hydrogenevolution transformation

\section{6 其他}

传统意义上, 苄位亚甲基 $\mathrm{C}_{\mathrm{sp} 3}-\mathrm{H}$ 键选择性氧化成 醛酮需要重金属的参与并使用当量的氧化剂. 而无金属 参与的有机小分子催化往往具有很强的底物限制性. 近 年来, 可见光催化氧化手段的蓬勃发展为该方向注入了 新鲜血液, 为芐位 $\mathrm{C}_{\mathrm{sp} 3}-\mathrm{H}$ 键选择性氧化在精细品化学 中的应用提供了一条崭新的思路.

2012 年, Togo 课题组 ${ }^{[29]}$ 利用 $\mathrm{KBr}$ 和过硫酸氢钾, 通 过㩲氢和直接氧化过程, 分别在热/光氧化条件下实现 了多种贫电子芳基酮类化合物的高效构建(图 13).

2014 年, 福州大学光催化物理研究所首次利用无 金属参与的 $\mathrm{C}_{3} \mathrm{~N}_{3} \mathrm{~S}_{3}$ 聚合物在可见光照射下实现了茮醇 到苯甲醛的选择性高效转化, 通过对 $\mathrm{rGO} / \mathrm{C}_{3} \mathrm{~N}_{3} \mathrm{~S}_{3}$ 夹心 结构进一步修饰可以实现更高效能的转化(图 14) ${ }^{[30]}$.

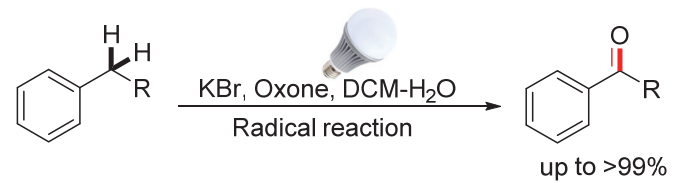

图 13 以 $\mathrm{KBr}$ 为氧化剂实现茮位直接氧化

Figure 13 Benzylic oxidation of alkylarenes using $\mathrm{KBr}$

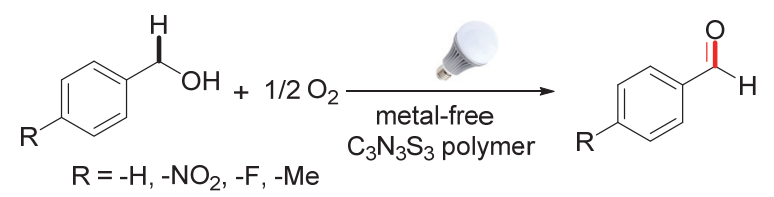

图 14 无金属参与的苄醇选择性光催化氧化

Figure 14 Metal-free catalysts for selective photocatalytic oxidation of benzylic alcohols

2015 年, Wolf 课题组 ${ }^{[31]}$ 利用核黄素四乙酸酯 RFT 和 $\mathrm{Sc}(\mathrm{OTf})_{3}$ 催化体系实现了贫电子烷基苯和苄醇苄位 $\mathrm{C}_{\mathrm{sp} 3}-\mathrm{H}$ 键的需氧氧化, 并认为路易斯酸在调节核黄素 氧化还原电位方面起着重要的作用(图 15).

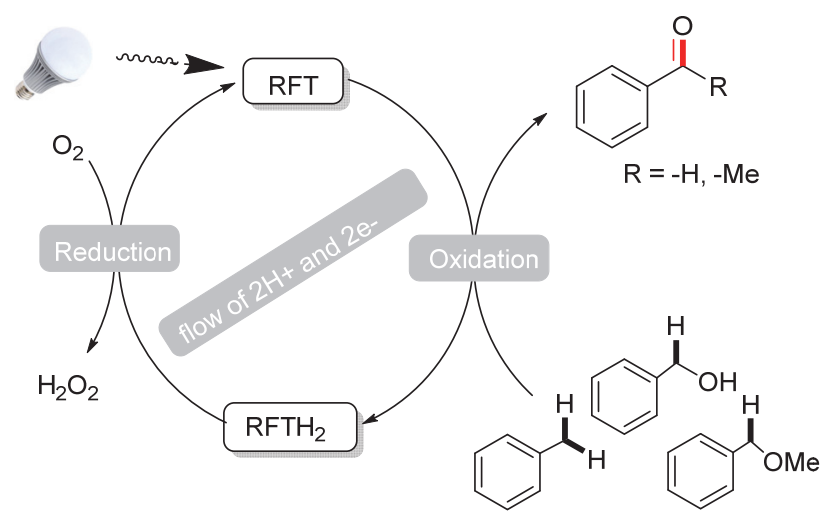

图 15 RFT 催化不同类型底物的需氧氧化

Figure 15 Aerobic oxidation of various organic substrates

2015 年, Goldberg 教授课题组 ${ }^{[32]}$ 利用 $\mathrm{Mn}^{\mathrm{III}}$ 络合物, 在可见光诱导下, 实现了茮位 $\mathrm{C}$ - $\mathrm{H}$ 需氧氧化历程(图 16). 他们通过 $\mathrm{X}$ 射线单晶衍生对基态催化剂的空间结 构进行了表征, 并认为物种 $\left[\mathrm{Mn}^{\mathrm{III}}\left(\mathrm{H}_{2} \mathrm{O}\right)\left(\mathrm{TBP}_{8}-\right.\right.$

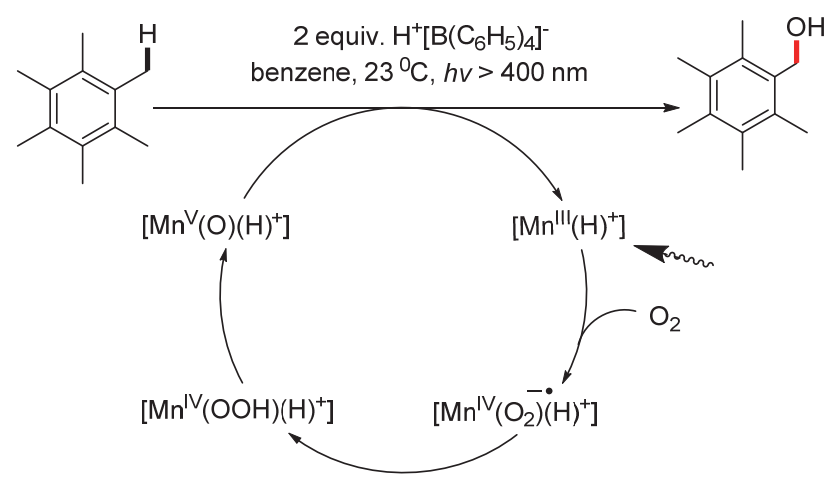

图 16 催化氧化循环示意图

Figure 16 Catalytic cycle for the oxidation of HMB 
$\mathrm{Cz}(\mathrm{H}))]^{+}$质子化的数目和位置对于调控催化物种与氧气 反应的活性具有重要影响.

2015 年, 雷爱文教授课题组 ${ }^{[33]}$ 通过光化学合成的 手段, 以绿色、安全、环境友好的氧气为终端氧化剂, 实 现了茮位 $\mathrm{C}_{\mathrm{sp} 3}-\mathrm{H}$ 键到酮类化合物的转变(图 17). 此方 法也适用于两个芳环均有取代基的底物, 丰富了反应的 应用范围. 自由基抑制实验和间歇光照实验表明该反应 可能是经光引发的自由基反应历程. 同时, ${ }^{18} \mathrm{O}$ 同位素标 记实验证明羰基氧来源于氧气.

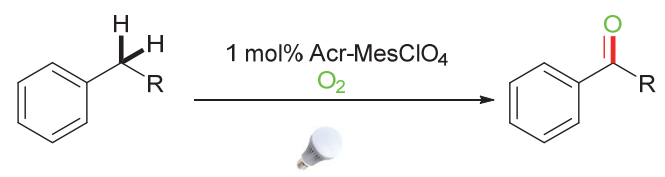

图 17 光致氧化茮位 $\mathrm{C}_{\mathrm{sp} 3}-\mathrm{H}$ 键制备酮类化合物

Figure 17 Photooxygenation of benzylic $\mathrm{C}_{\mathrm{sp} 3}-\mathrm{H}$ to ketones

\section{7 小结与展望}

对于可见光介导的, 涉及单电子转移过程的茮位 $\mathrm{C}_{\mathrm{sp} 3}-\mathrm{H}$ 键活化, 反应形式灵活多变, 展现出独特的合 成优势, 在合成形式上实现了简单、理想的化学成键模 式. 这些研究为有机合成方法学的探索注入了薮新的活 力, 展示了这一领域较为广阔的研究潜力和应用前景. 但也可以看出, 这类反应底物限制性较大, 机理研究尚 不明确. 目前, 对可见光促进的苄位 $\mathrm{C}_{\mathrm{sp} 3}-\mathrm{H}$ 键活化的 研究还处于起步阶段, 仍需要更多的研究来揭示其内部 规律以及进一步拓展其在合成中的应用.

\section{作者简介}

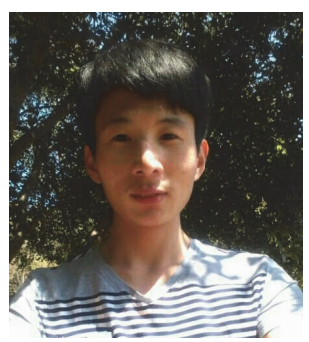

裴朋昆, 于 2015 年进入武汉大学攻读硕士学位, 师从雷 爱文教授, 主要研究领域为可见光催化的氧化偶联反应.

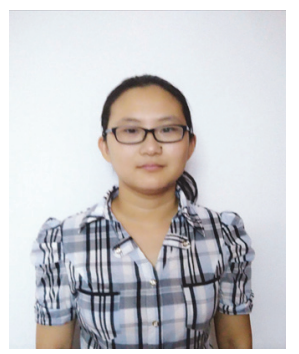

张凡, 自 2014 年起就读于武汉大学, 攻读理学学士学位, 指导老师为雷爱文教授. 主要研究领域为过渡金属催化和光 催化的碳-碳、碳一杂原子成键方法学研究.

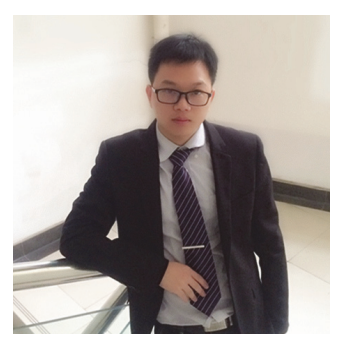

易红, 2012 年毕业于武汉大学, 获得理学学士学位, 同年 于武汉大学攻读博士, 指导老师为雷爱文教授. 主要研究领 域为金属催化, 光催化的偶联反应及反应机理研究.

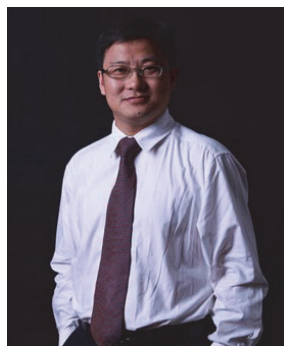

雷爱文, 教授. 1995 年本科毕业于安徽淮北煤炭师范学院, 2000 年于中国科学院上海有机化学研究所获理学博士学位, 师从陆熙炎院士. 2000 年至 2003 年间, 在美国宾夕法尼亚州 州立大学张绪穆教授课题组从事博士后研究. 2003 年至 2005 年在美国斯坦福大学 James P. Collman 教授课题组从事研究助 理工作. 2005 年回国到武汉大学化学与分子科学学院全职任 教. 2010 年获得国家杰出青年基金. 2014 年入选教育部长江学 者特聘教授. 现主要从事有机小分子合成方法学以及化学反 应活性中间体和反应动力学的机理研究.

\section{References}

[1] (a) Vanjari, R.; Singh, K. N. Chem. Soc. Rev. 2015, 44, 8062; (b) Zhang, J.; Lu, Q.-Q.; Liu, C.; Lei, A.-W. Chin. J. Org. Chem. 2015, 35(4), 743. (张剑, 陆庆全, 刘超, 雷爱文, 有机化学, 2015, 35(4), 743.); (c) Huang, Z.; Tang, S.; Lei, A. Sci. Bull. 2015, 60, 1391.

[2] Prier, C. K.; Rankic, D. A.; MacMillan, D. W. Chem. Rev. 2013, 113, 5322.

[3] Koike, T.; Akita, M. Inorg. Chem. Front. 2014, 1, 562.

[4] Ravelli, D.; Protti, S.; Fagnoni, M. Chem. Rev. 2016, 116, 9850.

[5] (a) Xi, Y.; Yi, H.; Lei, A.-W. Org. Biomol. Chem. 2013, 11, 2387; (b) Zeng, T.-T.; Xuan, J.; Chen, J.-R.; Lu, L.-Q.; Xiao, W.-J. Imaging Science and Photochemistry 2014, 32, 415. (曾婷婷, 宣俊, 陈加 荣, 陆良秋, 肖文精, 影像科学与光化学, 2014, 32, 415.)

[6] Hopkinson, M. N.; Sahoo, B.; Li, J.-L.; Glorius, F. Chem. Eur. J. 2014, 20, 3874.

[7] Nicewicz, D. A.; Nguyen, T. M. ACS Catal. 2014, 4, 355.

[8] Hari, D. P.; Konig, B. Angew. Chem. Int. Ed. 2013, 52, 4734

[9] Yoon, T. P.; Ischay, M. A.; Du, J. Nat. Chem. 2010, 2, 527.

[10] Xuan, J.; Xiao, W.-J. Angew. Chem. Int. Ed. 2012, 51, 6828.

[11] Xia, J.-B.; Zhu, C.; Chen, C. J. Am. Chem. Soc. 2013, 135, 17494.

[12] Xia, J.-B.; Zhu, C.; Chen, C. Chem. Commun. 2014, 50, 11701.

[13] Qvortrup, K.; Rankic, D. A.; MacMillan, D. W. C. J. Am. Chem. Soc. 2014, 136, 626.

[14] Hager, D.; MacMillan, D. W. C. J. Am. Chem. Soc. 2014, 136, 16986.

[15] Rabet, P. T. G.; Fumagalli, G.; Boyd, S.; Greaney, M. F. Org. Lett. 2016, $18,1646$.

[16] Hou, T.-Y.; Lu, P.; Li, P.-X. Tetrahedron Lett. 2016, 57, 2273.

[17] Lu, P.; Hou, T.-Y.; Gu, X.-Y.; Li, P.-X. Org. Lett. 2015, 17, 1954.

[18] Pandey, G.; Laha, R.; Singh, D. J. Org. Chem. 2016, 81, 7161.

[19] Zou, Y.-Q.; Lu, L.-Q.; Fu, L.; Chang, N.-J.; Rong, J.; Chen, J.-R.; Xiao, W.-J. Angew. Chem. Int. Ed. 2011, 50, 7171

[20] Liu, Q.; Li, Y.-N.; Zhang, H.-H.; Chen, B.; Tung, C.-H.; Wu, L.-Z. 
Chem. Eur. J. 2012, 18, 620.

[21] Zhong, J.-J.; Meng, Q.-Y.; Wang, G.-X.; Liu, Q.; Chen, B.; Feng, K.; Tung, C.-H.; Wu, L.-Z. Chem. Eur. J. 2013, 19, 6443.

[22] Gao, X.-W.; Meng, Q.-Y.; Li, J.-X.; Zhong, J.-J.; Lei, T.; Li, X.-B.; Tung, C.-H.; Wu, L.-Z. ACS Catal. 2015, 2391.

[23] Zheng, Y. W.; Chen, B.; Ye, P.; Feng, K.; Wang, W.; Meng, Q. Y.; Wu, L.-Z.; Tung, C.-H. J. Am. Chem. Soc. 2016, 138, 10080

[24] Zhong, J.-J.; Meng, Q.-Y.; Liu, B.; Li, X.-B.; Gao, X.-W.; Lei, T.; Wu, C.-J.; Li, Z.-J.; Tung, C.-H.; Wu, L.-Z. Org. Lett. 2014, 16, 1988.

[25] Zhong, J.-J.; Wu, C.-J.; Meng, Q.-Y.; Gao, X.-W.; Lei, T.; Tung, C.-H.; Wu, L.-Z. Adv. Synth. Catal. 2014, 356, 2846.

[26] Meng, Q.-Y.; Zhong, J.-J.; Liu, Q.; Gao, X.-W.; Zhang, H.-H.; Lei, T.; Li, Z.-J.; Feng, K.; Chen, B.; Tung, C.-H.; Wu, L.-Z. J. Am.
Chem. Soc. 2013, 135, 19052

[27] Ye, P.; Wang, D.-H.; Chen, B.; Meng, Q.-Y.; Tung, C.-H.; Wu, L.-Z. Sci. China. Chem. 2016, 59, 175.

[28] Xiang, M.; Meng, Q.-Y.; Li, J.-X.; Zheng, Y.-W.; Ye, C.; Li, Z.-J.; Chen, B.; Tung, C.-H.; Wu, L.-Z. Chem. Eur. J. 2015, 21, 18080.

[29] Moriyama, K.; Takemura, M.; Togo, H. Org. Lett. 2012, 14, 2414.

[30] Xu, J.; Luo, L.; Xiao, G.; Zhang, Z.; Lin, H.; Wang, X.; Long, J. ACS Catal. 2014, 4, 3302.

[31] Muhldorf, B.; Wolf, R. Chem. Commun. 2015, 51, 8425.

[32] Neu, H. M.; Jung, J.; Baglia, R. A.; Siegler, M. A.; Ohkubo, K.; Fukuzumi, S.; Goldberg, D. P. J. Am. Chem. Soc. 2015, 137, 4614.

[33] Yi, H.; Bian, C.; Hu, X.; Niu, L.; Lei, A. Chem. Commun. 2015, 51, 14046. 